\title{
KEYNOTE LECTURES
}

random phason is taken into account as the phason displacement parameter in the refinement. The paper demonstrates structure determination of quasicrystals in the light of recently developed techiniques.

[1] Yamamoto A., Takakura H., Ferroelectrics, 2004, 305, 223. [2] Takakura H., Shiono M., Sato T. J.,Yamamoto A.,Tsai A. P., Phys. Rev. Lett., 2001, 86, 236.

Keywords: quasicrystals, structure refinement, direct methods

\section{KN11.26}

Acta Cryst. (2005). A61, C3

Structure of Protein Assemblies by Comparative Modeling and Electron Microscopy

Andrej Sali ${ }^{\mathrm{a}}$, Maya Topf ${ }^{\mathrm{a}}$, Mathew L. Baker ${ }^{\mathrm{b}}$, Wah Chiu ${ }^{\mathrm{b}},{ }^{\mathrm{a}}$ University of California, San Francisco, CA. ${ }^{b}$ Baylor College of Medicine, Houston, TX. E-mail: sali@salilab.org

We explore structural characterization of protein assemblies by a combination of electron cryo-microscopy (cryoEM) and comparative protein structure modeling (1). Specifically, our method finds an optimal atomic model of a given assembly subunit and its position within an assembly by fitting alternative comparative models into a cryoEM map. The alternative models are calculated by MODELLER (2) from different sequence alignments between the modeled protein and its template structures. The fitting of these models into a cryoEM density map is performed by a new density fitting module of MODELLER (Mod-EM). Identification of the most accurate model is based on the correlation between the model accuracy and the quality of fit into the cryoEM density map. To quantify this correlation, we created a benchmark consisting of eight proteins of different structural folds with corresponding density maps simulated at five resolutions from 5 to $15 \AA$, with three noise levels each. Each of the proteins in the set was modeled based on 300 different alignments to their remotely related templates (12-32\% sequence identity), spanning the range from entirely inaccurate to essentially accurate alignments. The benchmark revealed that one of the most accurate models can usually be identified by the quality of its fit into the cryoEM density map, even for noisy maps at $15 \AA$ resolution. Therefore, a cryoEM density map can be helpful in improving the accuracy of a comparative model. Moreover, a pseudo-atomic model of a component in an assembly may be built better with comparative models of the native subunit sequences than with experimentally determined structures of their homologs.

[1] Topf M., Baker M.L., John B., Chiu W., Sali A., J. Struct. Biol.,in press. [2] Sali A., Blundell T.L., J. Mol. Biol.,1993, 234, 779-815.

Keywords: comparative modeling, electron cryo-microscopy, fitting

\section{KN12.26}

Acta Cryst. (2005). A61, C3

Quantum Mechanical Simulation of the Vibrational Properties of Garnets

Roberto Dovesi $^{\mathrm{a}}$, Roberto Orlando ${ }^{\mathrm{b}}$, Fabien Pascale ${ }^{\mathrm{c}}$, Javier F. Torres ${ }^{\mathrm{a}}$, ${ }^{\mathrm{a}}$ Dipartimento di Chimica IFM, Università di Torino. ${ }^{\mathrm{b}}$ Dipartimento di Scienze e Tecnologie Avanzate, Università del Piemonte Orientale. ${ }^{\mathrm{c}}$ Laboratoire de Pétrologie, Modélisation des Matériaux et Processus, Université Pierre et Marie Curie. E-mail: roberto.dovesi@unito.it

Structural, vibrational and electronic properties of pyrope, grossular and andradite have been simulated with the periodic $a b$ initio CRYSTAL [1] program, that adopts a local variational basis set ("Atomic Orbitals") to build the crystalline orbitals. An all-electron basis and the B3LYP hamiltonian have been used.

The full spectrum at the $\Gamma$ point (97 frequencies) [2] and the IR intensities have been evaluated, along with the symmetry of the modes, which is automatically determined. The eigenvectors of the dynamical matrix have been analyzed with different tools, including direct inspection, isotopic substitution, animations; classificationinterpretation questions raised by previous studies are discussed.

The 17 IR and 25 RAMAN active modes are compared with available experimental data [3], [4]. The agreement is excellent in most of the cases $\left(6-8 \mathrm{~cm}^{-1}\right.$ the mean absolute difference).

[1] Saunders V.R., Dovesi R., Roetti C., Orlando R., Zicovich-Wilson C., Harison N.H. Doll K., Civalleri B., Bush I.J., D'Arco Ph., Llunell M., CRYSTAL2003 user's manual. University of Torino, Torino, 2003. [2] Pascale F., Zicovich-Wilson C., Orlando R., Dovesi R. J., Phys. Chem., 2005, in press [3] Kolesov B., Geiger C., Phys. Chem. Min, 2000, 27, 645. [4] Hofmeister A., Chopelas A., Phys. Chem. Min, 1991, 17, 503.

Keywords: ab-initio calculations, garnets, vibrational frequencies

KN13.26

Acta Cryst. (2005). A61, C3

In Situ X-ray Study of Hydrothermally Prepared Titanates and Silicotitanates

Abraham Clearfield, Akhilesh Tripathi, Dmitri Medvedev, Department of Chemistry, Texas A\&M University, College Station, TX, USA. E-mail: clearfield@mail.chem.tamu.edu

In a typical ex-situ hydrothermal or solvothermal reaction the investigator knows what is input and what the end result is but has no experimental evidence of what transpires in between. In situ methods, whether using X-ray, NMR, IR or other techniques aims to follow the reaction through this in-between stage. For crystal growth studies, insitu X-ray diffraction is eminently satisfactory. We will describe our crystal growth studies of titanium silicate phases and the ion exchange mechanism of $\mathrm{Cs}^{+}$ion uptake in their tunnel structures.

One of the most vexing problems facing the nuclear industry and countries with nuclear weapons is the safe disposal of the generated nuclear waste. Huge quantities of nuclear waste arising from weapons manufacture are stored at the Hanford and Savannah River sites. The general method of remediation involves the removal of Cs-137, Sr-90 and actinides from a huge quantity of salts, principally $\mathrm{NaNO}_{3}$, organics and complexing agents. It has been found that a sodium silicotitanate is able to remove $\mathrm{Cs}^{+}$selectively from the waste and certain sodium titanates remove $\mathrm{Sr}^{2+}$ and actinides. These compounds have been prepared by ex-situ hydrothermal methods. We have studied the in situ growth of these materials at the National Synchrotron Light Source, Brookhaven National Laboratory.* In addition we will describe the mechanism of ion exchange in the titanosilicate as observed by in situ methods and how the combination of these techniques coupled with an intimate knowledge of the structure of the solids is helping to solve the remediation process. In general, the in situ method allows the investigator to follow the nucleation and crystal growth or phase transformations occurring in hydrothermal reactions, and as a result of ion exchange reactions.

* In collaboration with Aaron J. Celestian, Department of Geosciences and John B. Parise and Jonathan Hanson, Department of Chemistry, SUNY Stony Brook, NY, USA.

[1] Medvedev D.M., Tripathi A, Clearfield A., Celestian A.J., Parise J.B., Hanson J., Chem. Mater., 2004, 16, 3659.

Keywords: hydrothermal synthesis, in situ reactions, in-situ powder diffraction

\section{KN14.26}

Acta Cryst. (2005). A61, C3-C4

\section{Catalysts of De Novo Disulfide Bond Formation}

Deborah Fass, Einav Gross, Nimrod Heldman, Elvira Vitu, Department of Structural Biology, Weizmann Institute of Science, Rehovot, Israel. E-mail: deborah.fass@weizmann.ac.il

Disulfide bonds are crucial for the folding and stability of many cell-surface and secreted proteins. In addition, disulfides can be used for redox control of protein activity. De novo disulfide bond formation by enzymes such as those that drive oxidative protein folding requires transfer of electrons from dithiols to non-thiol electron acceptors. We have determined the structures of representatives of the two known eukaryotic enzyme families that catalyze this process. These enzymes, Ero1 and Erv2, are flavoproteins that share similar structural and mechanistic features despite a lack of sequence similarity. In particular, our crystallographic and enzymological studies suggest that both enzymes operate by a "disulfide shuttle" mechanism, in which a dithiol motif on a mobile segment of each enzyme transfers electrons from cysteines in substrate proteins to the rigid active-site disulfide. In 


\section{KEYNOTE LECTURES}

both enzyme families, the shuttle disulfide determines the substrate specificity. Furthermore, both the Ero1 and Erv2 families can transfer electrons to a similar panel of electron acceptors. One striking difference between the two enzymes, however, is that Erol displays kinetic complexities not shared by Erv2. This difference will be discussed in light of the structures, a mechanistic model will be presented, and a possible physiological role for the complex behavior of Ero1 will be suggested.

Keywords: flavoenzymes, disulfides, enzyme mechanisms

KN15.26

Acta Cryst. (2005). A61, C4

Synthetic Crystallography

A. Guy Orpen, School of Chemistry, University of Bristol, Bristol BS8

1TS, United Kingdom. E-mail: guy.orpen@bris.ac.uk

The synthesis of new and designed crystal structures is part of a major strand of modern chemistry in which the focus has shifted from the analytical function of crystallography (the determination of crystal structures) to the synthetic. In synthetic crystallography, itself a branch of crystal engineering, a variety of means have been used in the attempt to plan and control the products and objectives of synthesis - the crystal structures.

Here strategies that might be adopted to achieve this synthetic goal are considered. In particular the utility of approaches based on supramolecular chemistry and molecular tectonics are examined. Progress is evaluated towards control of :

a. The composition of the final synthetic product - the crystal structure

b. The supramolecular synthon formed.

c. The periodic motif(s) formed.

d. The entire crystal structure.

Applications and properties of such "engineered" solids is of importance. One property of particular interest to chemists is reactivity - this at the heart of the history of crystal engineering and has received sustained attention from a relatively small number of groups, primarily in solid state organic and organometallic chemistry. The prospects for the application of supramolecular crystals in synthesis and the opportunity to exploit the unusual constraints they impose on molecular reactivity are considered.

Keywords: crystal engineering, supramolecular crystallography, inorganic solid-state chemistry

\section{KN16.26}

Acta Cryst. (2005). A61, C4

A Perspective on the Crystal Structures of High Pressure Elements and their Properties

John S. Tse, Department of Physics and Engineering Physics University of Saskatchewan, Saskatoon, Canada S7N 5E2. Steacie Institute for Molecular Sciences National Research Council of Canada, Ottawa, Canada K1A 0R6. E-mail: John.Tse@usask.ca

Recent advancements in instrumentations and improvements in structural refinement techniques have led to the characterization of many structures of solids at high pressures. Some of the structure types discovered is novel and not seen in solids under ambient conditions. Even for elemental solids, particularly at intermediate pressure regime, it was found that instead of adopting simple closepack structures, open and complex structures, modulated structures or incommensurately modulated structures were often observed. These observations challenge the conventional concept of chemical bonding for solids and provide a fertile ground for the investigation of new physical phenomena in materials under high pressure. In this presentation, high pressure structures and transformations on specific elemental solids are illustrated and discussed. The purpose is to develop a conceptual framework for the description of the structures and the understanding of the nature of chemical bonding at high pressure. It is shown that the distinct electronic structure and structural features are related to other unusual properties such as superconductivity.

Keywords: high-pressure structures, electronic structure calculations, chemical bonding theory
KN17.27

Acta Cryst. (2005). A61, C4

Some New Insights in to the Mechanisms of Fullerene and Nanotube Formation

Harold Kroto, Chemistry Department, Florida State University, FL, USA. Chemistry Department, University of Sussex, UK. E-mail: kroto@sussex.ac.uk

In 1985 Buckminsterfullerene (the third allotropic form of carbon) was discovered during experiments designed to unravel the chemistry in red giant carbon stars. The molecule has now come down to Earth giving rise to the Fullerenes, a family of pure carbon cage molecules with fascinating properties which promise exciting new developments in 21st Century Materials Science and Technology. Fullerene molecules and their elongated nanotube (buckytube) cousins are now the subject of intense study as they promise to play major roles in almost every possible area of future technology from medicine and molecular electronics to civil engineering. However the mechanisms whereby various types of nanostructures assemble are still very poorly understood. Over the last decade or so, we have examined a wide range of methods for nanotube formation and from these studies some interesting new insights have been gained - especially with regard to metal catalysed nanostructure formation.

Keywords: fullerene, nanotube, $\mathbf{C 6 0}$

\section{KN18.27}

Acta Cryst. (2005). A61, C4

Protein Kinase Inhibition and Substrate Recognition

Louise N. Johnson, Laboratory of Molecular Biophysics, Biochemistry Department, University of Oxford. E-mail:

louise.johnson@biop.ox.ac.uk

Protein kinases are key components of cell signalling pathways. Defects in these processes lead to diseases such as cancer, diabetes and arthritis and hence protein kinases have become targets for drug design and therapy. We recently reviewed progress in this field with reference to kinase inhibitors that are in clinical trials or in the clinic and for which structural information is available[1]. In this talk I shall review some of our work with reference to cell cycle protein kinases [2] and I shall expand the discussion to consider wider aspects of substrate recognition with reference to $\mathrm{CDK} 2 /$ cyclin $\mathrm{A}, \mathrm{CDK} 2 /$ cyclin $\mathrm{E}$ [3], CDK7 [4] and polo-like kinase[5].

[1] Noble M.E., Endicott J.A., Johnson L.N., Science, 2004, 303, 1800. [2] Davies T.G., Bentley J., et al., Nature Structural Biology, 2002, 9, 745. [3] Honda R., Lowe E.D., Dubinina E., Skamnaki V., Cook A., Brown N.R., Johnson L.N., EMBO J., 2005, 24, 452. [4] Lolli G., Lowe E.D., Brown N.R., Johnson L.N., Structure (Camb), 2004, 12, 2067. [5] Cheng K.-Y., Lowe E.D., Sinclair J., Nigg E.A., Johnson, L.N., EMBO J., 2003, 22, 5757.

Keywords: protein kinases, inhibitors, cell cycle

\section{KN19.27}

Acta Cryst. (2005). A61, C4-C5

Crystallography in Inorganic Solid-State Chemistry

James A. Ibers, Department of Chemistry, Northwestern University, Evanston, IL 60208-3113, U.S.A. E-mail: ibers@chem.northwestern.edu

The pervasive importance of crystallography, particularly results obtained from single-crystal studies, in inorganic solid-state chemistry will be explored through a number of examples of metal chalcogenide systems. These examples will also illustrate the importance of other physical measurements in the characterization of such compounds. Among such examples, the $\mathrm{AMM}^{\prime} \mathrm{Q}_{3}$ system $(\mathrm{A}=$ alkali metal; $\mathrm{M}=\mathrm{f}$ element; $\mathrm{M}^{\prime}=\mathrm{d}$-element; $\mathrm{Q}=\mathrm{S}, \mathrm{Se}$, or Te) will be used to emphasize the importance of concomitant optical and magnetic measurements and the compound $\mathrm{RbVSe}_{2}$ will serve to illustrate the importance of theoretical calculations.

There are also pitfalls and difficulties in the applications of crystallography to solid-state systems. The determination of chemical composition through the refinement of site occupancies is one of the most highly abused areas. Some aspects of the refinement of site occupancies will be explored through the NaLiM'S ${ }_{2}$ series and the $\mathrm{Er}_{2}$ 